\title{
A Predictive Model-Based Decision Support System for Diabetes Patient Empowerment
}

\author{
Dietmar GLACHS ${ }^{\mathrm{a}, 1}$, Tuncay NAMLI ${ }^{\mathrm{b}}$, Felix STROHMEIER ${ }^{\mathrm{a}}$, Gustavo RODRÍGUEZ \\ SUÁREZ ${ }^{\mathrm{c}}$, Michel SLUIS ${ }^{\mathrm{d}}$, Javier DELGADO-LISTA ${ }^{\mathrm{e}}$, Jacob K. SONT ${ }^{\mathrm{f}}$, Albert A. \\ DE GRAAF ${ }^{\mathrm{g}}$, Eckhard SALZSIEDER ${ }^{\mathrm{h}}$ and Lutz VOGT ${ }^{\mathrm{h}}$

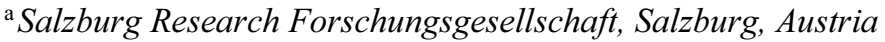 \\ ${ }^{\mathrm{b}}$ Software Research \& Development and Consultancy Corp., Ankara, Turkey \\ ${ }^{\mathrm{c}}$ iHealthLabs EUROPE SAS, Paris, France \\ ${ }^{\mathrm{d}}$ Prime Vision, Delft, Netherlands \\ e Servicio Andaluz de Salud or Andalusian Health Service, Cordoba, Spain \\ ${ }^{\mathrm{f}}$ Leiden University Medical Center, Leiden, Netherlands \\ ${ }^{\mathrm{g}}$ Dep. Risk Analysis for Products in Development, TNO, Utrecht, Netherlands \\ h Institute of Diabetes "Gerhard Katsch" Karlsburg, Karlsburg, Germany
}

\begin{abstract}
The main objective of POWER2DM is to develop and validate a personalized self-management support system (SMSS) for T1 and T2 diabetes patients that combines and integrates i) a decision support system (DSS) based on leading European predictive personalized models for diabetes interlinked with predictive computer models, ii) automated e-coaching functionalities based on Behavioral Change Theories, and iii) real-time Personal Data processing and interpretation. The SMSS offers a guided workflow based on treatment goals and activities where a periodic review evaluates the patients progress and provides detailed feedback on how to improve towards a healthier, diabetes appropriate lifestyle.
\end{abstract}

Keywords. diabetes, self-management, predictive modeling, care plan management

\section{Introduction}

Diabetes increasingly leads to societal challenges in Europe and across the world. The prevalence of suffering this chronic disease has increased markedly over the last 30 years. Currently, $9.3 \%$ or 463 million people live with diabetes and the IDF estimates 578 million people suffering diabetes in 2030 [1]. Diabetes and its complications are treated with medication and regular screening, in addition to diet and exercises. However, despite the increasing availability of solutions to monitor and control blood glucose levels, considered a key strategy to prevent diabetic complications, only a minority of diabetic patients currently reach their target of glycemic control, mostly due to an unhealthy lifestyle. Adopting and maintaining a healthy lifestyle in combination with psychological well-being is the key objective in achieving treatment goals and for

\footnotetext{
${ }^{1}$ Corresponding Author, Dietmar Glachs, Salzburg Research Forschungsgesellschaft m.b.H., JakobHaringer-Straße 5/3, 5020 Salzburg, Austria, E-mail: dietmar.glachs@salzburgresearch.at
} 
preventing diabetes complications [2]. Patient empowerment [3] involves patients to a greater extent in their own healthcare process and disease management becomes an integrated part of their daily life. For this, accurate information and Communication Technologies play a key role in better management of diabetes based on a patient's need, desires, skill-level and available devices. Self-management interventions result in improvement in knowledge and understanding of diabetes, health behaviours and selfefficacy [4], HbA1c [5], body weight [6], and complications [7]. Moreover, multiple lifestyle interventions provide improvements on cardiovascular risk modulators such us BMI and blood pressure [8].

To support diabetes patients, the POWER2DM project [9] developed a personalized Self-Management Support System (SMSS) including i) a Decision Support System (DSS) based on leading predictive personalized models for diabetes prediction, ii) automated e-coaching functionalities based on Behavioral Change theories and iii) realtime Personal Data processing and interpretation including Just-In-Time interventions.

\section{Method}

The main objective of the SMSS is to support the patient when coping with his/her chronic disease. For this the SMSS implements the personalised Action Plan as a guided workflow as an iterative cycle, where patients adopt long term medical treatment goals into concrete self-management goals and subsequently plan short term actions suitable for achieving his or her personal goals. This workflow is first initiated based on a KADIS ${ }^{\circledR}[10]$ fingerprint, a 3-day data collection phase used to perform simulations, and risk analysis based on predictive risk score models such as the AVANCE Cardiovascular, AVANCE Kidney risk engine [11], the UKPDS [12] risk engine or the PMOT1D risk score [13]. By using the Shared Decision-Making Application (SDMA) together with the healthcare professional, the long-term treatment plan including treatment goals is created based on the predictions.

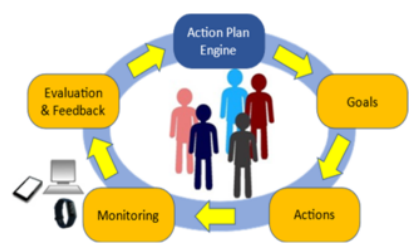

Figure 1. Patient Empowerment Workflow

Given the time limits per patient for healthcare professionals, treatment plans usually span longer periods of time. Hence, patients are encouraged to adopt treatment goals into personal goals and subsequently to plan activities he or she wants to accomplish within a shorter period of time. These short-term activities help to adhere to medical treatment plans, e. g. measuring glucose values, and also supports the accomplishment of personal and treatment goals. This action plan workflow has been introduced in [10] and comprises the following steps as depicted in Figure 1.

- Specify Values \& Goals: First, the patient specifies his or her self-management goals. These goals are still related to the medical treatment plan but reflect the patient's personal targets and values. Support is provided by the Value Compass, a tool reflecting on the importance of personal values in different life areas. 
- Plan Actions: Having self-management goals in mind, the patient creates his or her personal action plan for the next week and specifies tasks or activities suitable for achieving these goals. Tasks like meal intake, medication administration, glucose measurement or activities such as physical exercises are planned with a calendar. The duration of the personal action plan is not predefined, however, the patient's adherence to short term plans is typically higher compared to long-term plans.

- Monitoring: In everyday life, the scheduled tasks and activities are reported as calendar events to the patient who is forced to accomplish the planned tasks and activities. Integrated devices support monitoring of activities such as measuring blood glucose, blood pressure or body weight. Planned activities like physical exercises, meal intake or medication administration are recorded manually. Finally, it is possible to keep diaries regarding stress level, sleep quality, mood or (categorized) problems. The SMSS uses the recordings and provides Just-InTime Adaptive Interventions (JITAI) in agreement with the short and long-term goals of the patient.

- Evaluation \& Feedback: The system provides feedback on the patient's action plan coverage. Observations are related to tasks or activities and subsequently to self-management goals. The system computes performance results and provides interventions in various contexts. Interventions include recommendations based on national guidelines, tips for improving selfmanagement activities or tips for coping with daily (sleep or stress) problems. Motivational feedback messages support positive reinforcement [14], particularly upon successful completion of all tasks and activities in the review period and strengthens the patient's awareness and confidence in his or her selfmanagement activities, thus empowering the patients over time. In [15], a more detailed description of the intervention mechanisms is provided.

Each workflow cycle is finished with the evaluation of the performance and interventions provided in different contexts, such as tips for improving self-management activities, recommendations based on national guidelines, tips for coping with daily problems (e.g., sleep problems or stress) or motivational messages to support positive reinforcement [16], particularly when the patient has successfully completed all his activities in the review period. Evaluation also includes visits with health care professionals who then have, by means of the SDMA, insights to the collected data and can adopt the long-term medical treatment plan by taking new risk scores into account.

\section{Results}

Figure 2 illustrates the conceptual design of the Self-Management Support System (SMSS) consisting of SMSS web and mobile applications to be used by Type 1 and Type 2 diabetes patients and the Shared Decision-Making Application (SDMA) for joint use by patients and healthcare professionals. The User and Privacy Management provides the single sign-on page for all SMSS applications, the management of patients, users profiles and the user's privacy policies. A secure OAuth token is mandatory when using SMSS services. 
Both the SMSS Mobile and the SMSS Web Application support the patient with the self-management workflow. They allow collecting data, manually or with integrated devices, show the periodic review and also provide JITAI interventions to the user. The SMSS Web Application complements the mobile application and additionally supports the management of personal goals and allows the identification of barriers and evaluates the patient's goal adherence by providing evaluation feedback, e.g., motivational messages and tips for fostering or improving self-management activities.

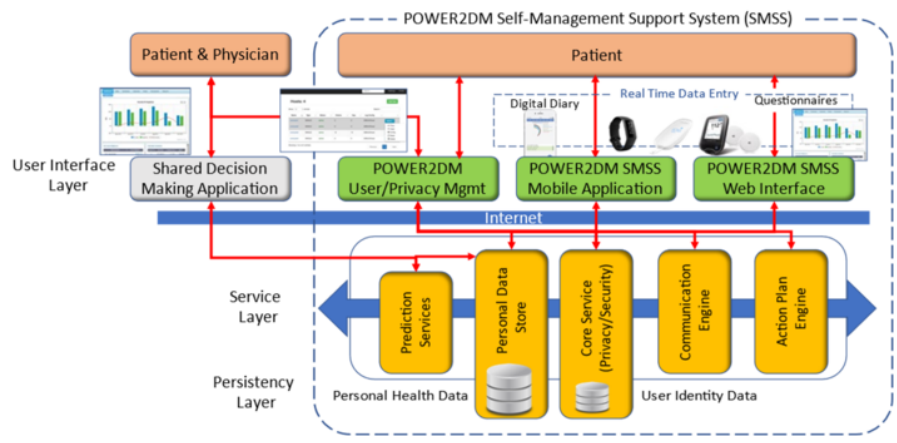

Figure 2. System Architecture - POWER2DM system

Each of the applications and components builds on the common data model and data exchange mechanisms provided by HL7 FHIR as a base standard (HL7 FHIR DSTU 2) [17]. This allows reusing even single components with current and future HL7 based applications. As shown in Figure 2, the main components of the SMSS are as follows:

- Personal Data Store: A fully FHIR compliant and secure data repository providing the personal health records. Data access is available solely with the FHIR API and requires an OAuth authorization token for secure access. The token is verified with the Privacy and Security service.

- Core Services: Provides core functionality like authentication, user identity and authorization management and audit logging.

- Prediction Services: Integrates simulation and risk score models, provides services for computing short-, mid- and long-term predictions as well as risk scores to other components, especially SDMA.

- Action Plan Engine: Provides interventions based on the collected data, computes the patient's adherence to his/her personal goals and values and provides (motivational) interventions. Provides workflow logic to applications.

\section{Conclusion}

The project conducted an initial feasibility study (FS) in two clinical centers: i) Leiden University Medical Center, NL and ii) Reina Sofia University Hospital, University of Cordoba, ES to prepare for a proper clinical trial. In each location, 5 patients and 3 healthcare practitioners (HCP) used the system prototype during 14 days. At the end of the FS, questionnaires for patients and HCP were filled out. The outcomes showed a good performance of the system in terms of functionality and expected benefits, and a good acceptability by both patients and HCP. No potential critical limitations in the system were found. Patients ranked the usability acceptable; for SMSS Web 7/10 in 
Spain and 6.3/10 in the Netherlands and for SMSS Mobile 5.8/10 and 6/10, resp. Average use of the system per day (23 mins in the Netherlands and $20 \mathrm{~min}$ in Spain) was perceived as adequate by patients. They qualified the instructions by the HCP and the feedback given by the system as supportive and helpful. HCP perceived the overall implementation of the system as good. Following the FS, the clinical trial included 230 diabetes patients (115 type-1 and 115 type-2), and ended in September 2020. Data analysis is still ongoing and will provide insights in the clinical and potential economic impacts of the system.

\section{References}

[1] IDF, Across the Globe. Available at https://diabetesatlas.org/en/sections/worldwide-toll-of-diabetes.html, Accessed Dec 23, 2020.

[2] Young-Hyman D, de Groot M, Hill-Briggs F, Gonzalez JS, Hood K, Peyrot M. Psychosocial Care for People With Diabetes: A Position Statement of the American Diabetes Association. Diabetes Care. 2016 Dec;39(12):2126-2140. doi: 10.2337/dc16-2053. Erratum in: Diabetes Care. 2017 Feb;40(2):287. Erratum in: Diabetes Care. 2017 May;40(5):726.

[3] Castro EM, Van Regenmortel T, Vanhaecht K, Sermeus W, Van Hecke A. Patient empowerment, patient participation and patient-centeredness in hospital care: A concept analysis based on a literature review. Patient Educ Couns. 2016 Dec;99(12):1923-1939.

[4] Wiebe DJ, Baker AC, Marino JA. Medical Systems and Patient-Provider Relationships. In: Delamater AM, Marrero DG, editors. Behavioral Diabetes: Social Ecological Perspectives for Pediatric and Adult Populations. Springer International Publishing. 2020; 463-78.

[5] Trief PM, Teresi JA, Eimicke JP, Shea S, Weinstock RS. Improvement in diabetes self-efficacy and glycaemic control using telemedicine in a sample of older, ethnically diverse individuals who have diabetes: the IDEATel project. Age Ageing. 2009 Mar;38(2):219-25.

[6] Colagiuri R, Eigenmann CA. A national consensus on outcomes and indicators for diabetes patient education. Diabet Med. 2009 Apr;26(4):442-6.

[7] Stratton IM, Adler AI, Neil HA, Matthews DR, Manley SE, Cull CA, Hadden D, Turner RC, Holman RR. Association of glycaemia with macrovascular and microvascular complications of type 2 diabetes (UKPDS 35): prospective observational study. BMJ. 2000 Aug 12;321(7258):405-12.

[8] Unick JL, Beavers D, Jakicic JM, Kitabchi AE, Knowler WC, Wadden TA, Wing RR; Look AHEAD Research Group. Effectiveness of lifestyle interventions for individuals with severe obesity and type 2 diabetes: results from the Look AHEAD trial. Diabetes Care. 2011 Oct;34(10):2152-7. doi: 10.2337/dc11-0874. Epub 2011 Aug 11.

[9] The POWER2DM project is funded by the European Union's Horizon 2020 research and innovation programme under grant agreement No 689444

[10] Salzsieder E, Vogt L, Kohnert KD, Heinke P, Augstein P. Model-based decision support in diabetes care. Comput Methods Programs Biomed. 2011 May;102(2):206-18

[11] Glachs D, Namli T, Jung O, Strohmeier F, Ploessnig M, Rodriguez G: FHIR Driven Self-Management Support System for Diabetes. In: Digital Personalized Health and Medicine. Proceedings of the 30th Medical Informatics Europe Conference. S. 2020. p.1291-1292.

[12] UKPDS Risk Engine Software, Available at https://www.dtu.ox.ac.uk/riskengine/, Accessed May 29, 2020 .

[13] Soedamah-Muthu SS, et al. (2014). Predicting major outcomes in type 1 diabetes: a model development and validation study. Diabetologia. 2014;57(11):2304-2314.

[14] Kengne AP, Patel A, Marre M, Travert F, Lievre M, Zoungas S, Chalmers J, Colagiuri S, Grobbee DE, Hamet P, Heller S, Neal B, Woodward M; ADVANCE Collaborative Group. Contemporary model for cardiovascular risk prediction in people with type 2 diabetes. Eur J Cardiovasc Prev Rehabil. 2011 Jun;18(3):393-8.

[15] Abraham C, Michie S. A taxonomy of behavior change techniques used in interventions. Health Psychol. 2008 May;27(3):379-87.

[16] Jung O, Glachs D, Strohmeier F, Mulrenin R, Huisman S, Smith I, van Keulen H, Sont J, Ploessnig M. Empowering Diabetes Patients with Interventions Based on Behaviour Change Techniques. Stud Health Technol Inform. 2019;260:154-161

[17] Elder JP, Ayala GX, Harris S. Theories and intervention approaches to health-behavior change in primary care. Am J Prev Med. 1999 Nov;17(4):275-84. 
Namli T, Köse O, POWER2DM D4.2 Personal Data Store Service Implementation, Available at http://power2dm.eu/wp-content/uploads/POWER2DM-D4.2.pdf, Access Jan 15, 2021. 